\title{
Jupyter Notebooks for Neutron Radiography Data Processing and Analysis
}

\author{
Jean-Christophe Bilheux ${ }^{\mathrm{a}}{ }^{*}$, Jiao Y. Y. Lin ${ }^{\mathrm{b}}$ and Hassina Z. Bilheux ${ }^{\mathrm{c}}$ \\ ${ }^{1}$ Neutron Scattering Division, Oak Ridge National Laboratory, Oak Ridge, TN 37831, USA \\ abilheuxjm@ornl.gov, 'linjiao@ornl.gov, 'bilheuxhn@ornl.gov
}

$\left(^{\star}\right)$ corresponding author

Keywords: Notebooks, Jupyter, Python, Neutron, Imaging, Analysis, Normalization

\begin{abstract}
Neutron radiography and computed tomography encompass a vibrant range of scientific applications, requiring advanced technique development and cutting-edge data processing and analysis software. We have developed an extensive portfolio of Python-based Jupyter notebooks that are custom-made for a specific experiment and sample geometry. These notebooks do not require any programming skills, although the code is accessible to programming experts if they wish to modify it. The notebooks are available on our analysis servers where the imaging data is also stored, preventing unnecessary and lengthy data transfer. This manuscript gives an overview of our efforts to empower the research community, that uses both the Spallation Neutron Source and High Flux Isotope Reactor imaging capabilities, to process and analyze their data in collaboration with our imaging team.
\end{abstract}

\section{Introduction}

Neutron radiography experiments impact a wide range of scientific areas such as materials science [1], energy [2], physics, engineering [3], archaeometry [4], plant physiology [5], geosciences [6], biology [7, 8], and chemistry. The High Flux Isotope Reactor (HFIR) CG-1D neutron imaging beamline [9] covers a broad range of scientific applications brought by scientists and engineers with different levels of expertise in image data analysis and programming. The facility is accessible at no cost through a peer review process if the visiting research team agrees to publish.

It is very challenging to develop a "one-shoe-fits-all" data processing and analysis software. A limited subset of the CG-1D research community performs data analysis without the help of the imaging team. The beamline is staffed with beamline scientists, data acquisition and software experts. This team works toward the development of intuitive data acquisition, processing and analysis tools that can be used by novice, intermediate and expert researchers. These users interact with our data processing and analysis software through the Python Jupyter notebooks. At this facility, most users rely on tools provided by the imaging team to process their data from normalization to 3-dimensional reconstruction, along with analysis of complex geometry samples. In order to improve the researcher's software experience and to make sure they are provided with adequate tools for their data processing and analysis, we implemented the procedure described undermentioned. Prior to arrival to the facility, we contact the principal investigator (PI) to discuss their data analysis needs as well as the level of software expertise of everyone in the PI's team. We already have many notebooks that can perform the most general needs of the users, but in case a team is coming with the need of a new tool, the pre-beamexperiment contact provides enough time to develop adequate (in terms of both algorithms and usability) tools. Upon arrival of the research team, we demonstrate the usage and provide a full step-by-step tutorial of each software. Finally, we follow up with the researchers after they have 
returned to their home institution, provide further software support, and get their feedback on our procedure and the software. In this article, we detail how we collaborate with researchers who come to use our neutron imaging beamline and demonstrate the advantages of using the Python Jupyter notebooks as a development and deployment environment.

\section{Experimental Planning}

While the beamline scientist often interacts with the research team as early as during the beam time proposal writing phase, data software discussions usually start when beam time has been granted. To optimize the visiting research team's experience at the beamline, a few key parameters need to be understood before their experimental beam time. First and foremost, we need to understand how the experiments can provide an answer to their scientific question. This leads to discussions about how the researchers plan to collect their data, what parameters need to be saved in the metadata and later extracted, and what information needs to be extracted from the radiographs and computed tomography (CT) scans. These early communications with the experimental researchers are critical to ensure data processing and analysis is in place either at the time the experiment is conducted or soon after. Second, our researchers have access to our previously developed Jupyter notebooks with tutorials from our imaging website [10]. Finally, the software experts spend time with the researchers at the beamline during the experiments to fully appreciate the complexity of the measurements and to confirm the data analysis strategy previously discussed.

\section{Jupyter Notebooks}

As defined by its official web site, the Jupyter Notebooks [11] is a versatile, open source web application that allows to create and share documents that contain live code, equations, visualizations and narrative texts. The notebooks are designed to intuitively develop and run codes. The Jupyter notebooks supports over 40 programming languages [12]. We chose to write code using the Python language which has an easy-to-learn and interpreted object-oriented programming language. The Jupyter interface is straightforward to use and thus, once familiar with the functionality of the interface, one can start coding inside a notebook using a web browser [13], see Fig. 1 (left). A notebook consists of a series of cells that each contains lines of code. Each cell can be run or rerun independently, pending the logic of the programming is respected. The code can be easily modified, such as changing an algorithm in a cell, without impacting the rest of the users.

In order to simplify the utilization of the notebooks, we implemented user interfaces (UI) that are launched from the notebook, as illustrated in Fig. 1 (right). This is possible because the thinlinc [14] connection to the SNS analysis servers is available for our users.

\section{How to Access the Notebooks}

Another benefit of using Jupyter notebooks is that they can be run from our analysis servers without having to install software on the researcher's computer. Since the imaging data reside on the servers, this prevents unnecessary transfer of large imaging data sets. Using a web browser and assuming the researcher has an account in our servers, they can log in. Once logged in the Linux server, they then click the jupyter imaging options on the "Applications and Software" dropdown menus. Within seconds, the user is switched to a new conda environment [15] which is a directory that contains all the installed python libraries/conda packages necessary to run the notebooks. The portfolio of the python notebooks is automatically imported into the user account, allowing them to modify the notebooks without modifying the original notebooks which are stored elsewhere, and the Jupyter server is started from the notebooks folder location. 

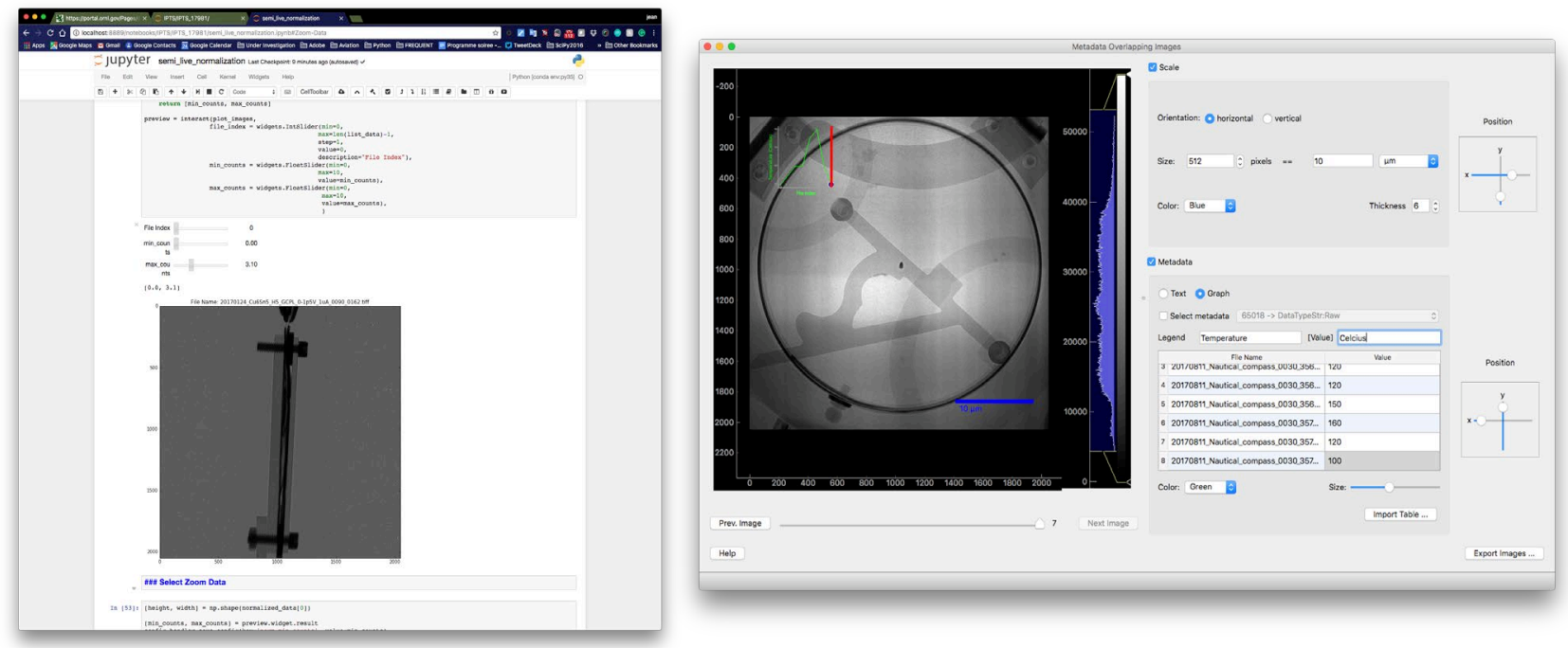

Figure 1. Left: An example of Python-based Jupyter notebook displayed using a web browser. Right: A user interface directly launched from a notebook.

The Jupyter home page shows a long list of various files and folders. The notebooks, files with extension ".ipynb”, are the only files most users will interact with. Advanced users can adventure inside the code itself, which can be found in the "_code" folder. Each notebook opens in a new tab and at its top is a link to a step-by-step tutorial (some with video tutorials, see Fig. 2) that explains the purpose of the code and how to run it. A parchment display summarizes the four important rules to remember to successfully run the notebooks, as illustrated in Fig. 2.

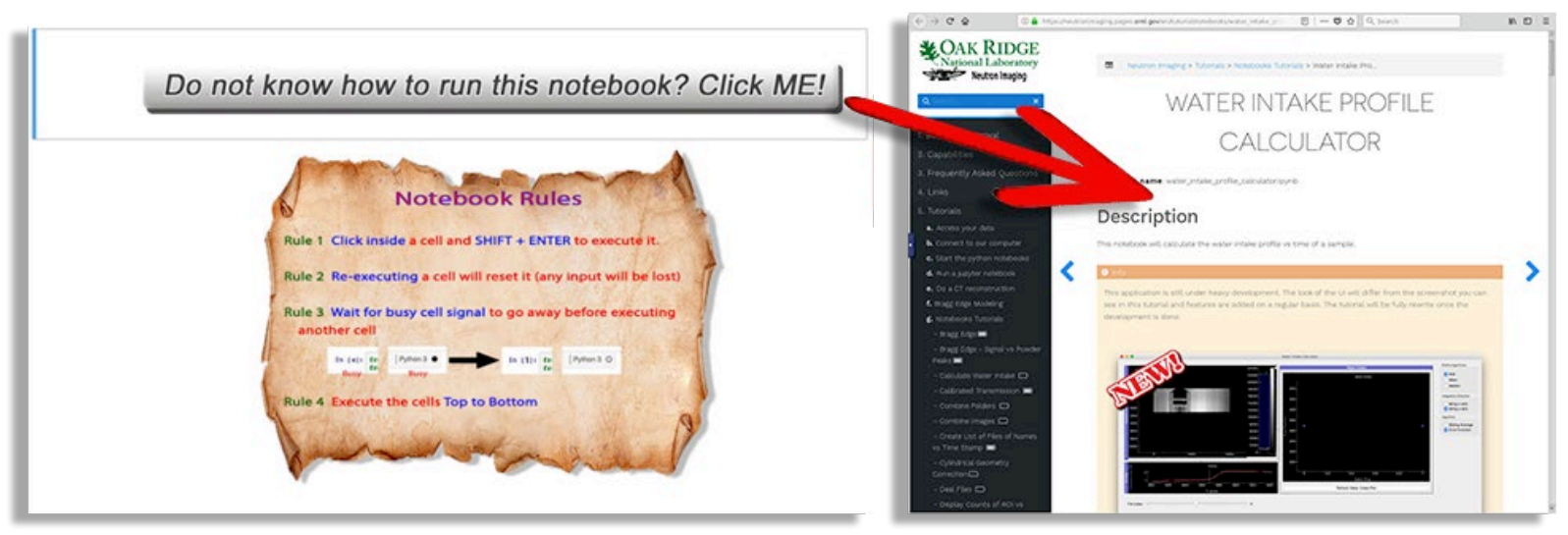

Figure 2. The top of each notebook provides a direct link (left) to a step-by-step tutorial (right) and a list of important rules (left) to execute the notebooks.

\section{Examples of Notebooks}

Within the past few years, we have developed a large library of Jupyter notebooks, all of which are available on our servers to all the CG-1D and the future VENUS (an imaging instrument at the Spallation Neutron Source) communities. These notebooks are custom-made for a specific experiment and sample geometry. A few examples are listed below to give a glance at the data processing and analysis software capability. 


\section{- Example 1: Binning of images based on metadata}

This notebook combines radiographs according to the temperature of the furnace saved in the metadata of each radiograph (Fig. 3). The notebook displays the entire temperature history of the experiment with the furnace file information as well as a time lapse of the corresponding radiographs (Fig. 3A). The code allows to select various binning options (by time, by temperature of the furnace, by number of imaging files). The principle of binning is similar to the event filtering for scattering instruments at the SNS [16]. Fig. 3B displays the binning that was applied in this example, and Fig. 3C shows the result of the binning with the created temperature steps.
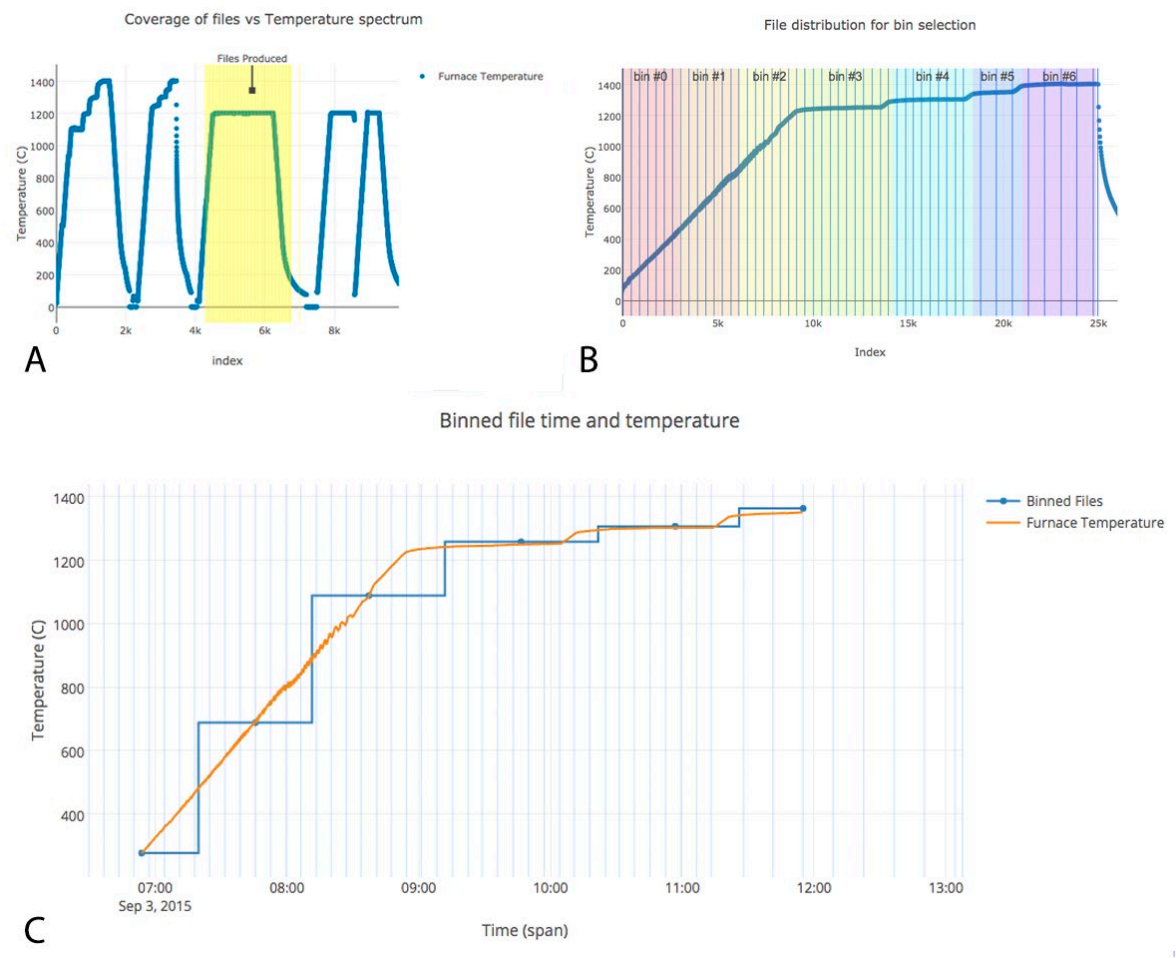

Figure 3. A: Furnace temperature information over entire experiment time (blue) and corresponding selected radiographs (yellow); B: color coded regions to show how the radiographs are binned; $C$ : binning result with average furnace temperature reported for each bin.

\section{- Example \#2: Water uptake in a soil sample}

This notebook calculates the water uptake velocity in a soil sample (Fig. 4). The most important notebook requirements for this research team were:

- avoid command line input,

- graphical selection of a region of interest,

- option to integrate region along $\mathrm{x}$ or $\mathrm{y}$ axis,

- be able to sort files using file name, or time stamp,

- be able to rename files (to improve sorting by file name),

- have the option to easily add algorithm used in the calculation of the front of the water uptake signal,

- export various profiles in ASCII file format. 
This example illustrates the complex requirements yet the versatility of a Jupyter notebook. Moreover, in collaboration with the research team, several algorithms that track the water front automatically were implemented and compared with each other. These implementations were straightforward and code-reuse were maximized.

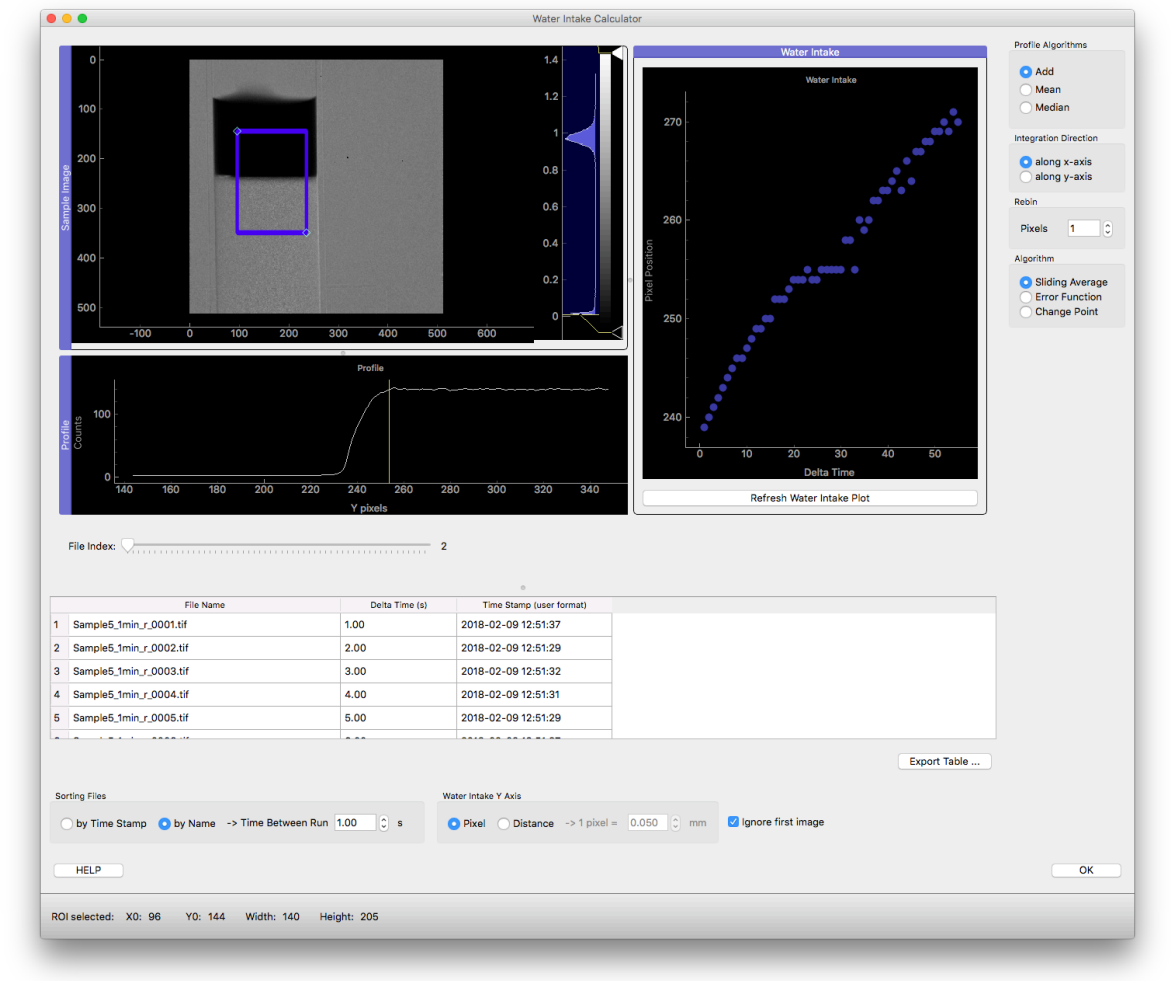

Figure 4. Water uptake notebook showing the profile of the water front versus time for a given region selected. (courtesy of Prof. Ed Perfect (University of Tennessee, Knoxville)).

\section{Conclusion}

Our team's approach to data processing and analysis is to provide easy-to-use code that is custom-made for a specific experiment and sample geometry, requires little to no programming expertise, and does not require complex software installation on the end-user's computer. Thus, we have successfully developed and deployed Python-based Jupyter notebooks that are available on our analysis servers. With these software capabilities, we can respond to the increasingly challenging and diverse research community that utilizes neutron imaging capabilities at the Spallation Neutron Source and the High Flux Isotope Reactor. The notebooks allow the most advanced users to modify the code directly, while less experienced users can take advantage of the graphical user interface. Step-by-step tutorials of all the notebooks are also available on our neutron imaging website to facilitate the use of the notebooks.

\section{Acknowledgements}

The authors would like to thank Prof. Ed Perfect and his team for their valuable input in improving our research community experience using the Jupyter notebooks. This research used resources at the High Flux Isotope Reactor and Spallation Neutron Source, DOE Office of Science User Facilities operated by the Oak Ridge National Laboratory. 
This manuscript has been authored [or, co-authored] by UT-Battelle, LLC, under contract DEAC05-00OR22725 with the US Department of Energy (DOE). The US government retains and the publisher, by accepting the article for publication, acknowledges that the US government retains a nonexclusive, paid-up, irrevocable, worldwide license to publish or reproduce the published form of this manuscript, or allow others to do so, for US government purposes. DOE will provide public access to these results of federally sponsored research in accordance with the DOE Public Access Plan (http://energy.gov/downloads/doe-public-access-plan).

\section{References}

[1] R. R. Dehoff, M. Kirka, W. Sames, H. Bilheux, A. Tremsin, L. Lowe, S. Babu. Site specific control of crystallographic grain orientation through electron beam additive manufacturing, Materials Science and Technology 31 (8) (2015) 931-938. https://doi.org/10.1179/1743284714Y.0000000734

[2] Y. Zhang, K. R. Chandran, H. Z. Bilheux, Imaging of the Li spatial distribution within v 2 o 5 cathode in a coin cell by neutron computed tomography, Journal of Power Sources 376 (2018) 125-130. https://doi.org/10.1016/j.jpowsour.2017.11.080

[3] D. J. Duke, C. E. Finney, A. Kastengren, K. Matusik, N. Sovis, L. Santodonato, H. Bilheux, D. Schmidt, C. Powell, T. Toops, High resolution x-ray and neutron computed tomography of an engine combustion network spray g gasoline injector, SAE International Journal of Fuels and Lubricants 10 (2017-01-0824) (2017) 328-343. https://doi.org/10.4271/2017-01-0824

[4] F. Salvemini, S. Olsen, V. Luzin, U. Garbe, J. Davis, T. Knowles, K. Sheedy, Neutron tomographic analysis: Material characterization of silver and electrum coins from the $6^{\text {th }}$ and $5^{\text {th }}$ centuries bce, Materials Characterization 118 (2016) 175-185.

https://doi.org/10.1016/j.matchar.2016.05.018

[5] M. Holz, A. Carminati, Y. Kuzyakov, Distribution of root exudates and mucilage in the rhizosphere: combining 14c imaging with neutron radiography, in: EGU General Assembly Conference Abstracts, Vol. 17, 2015.

[6] E. Perfect, C.-L. Cheng, M. Kang, H. Bilheux, J. Lamanna, M. Gragg, D. Wrigth, Neutron imaging of hydrogen-rich fluids in geomaterials and engineered porous media: A review, EarthScience Reviews 129 (2014) 120-135. https://doi.org/10.1016/j.earscirev.2013.11.012

[7] H. Z. Bilheux, J.-C. Bilheux, W. B. Bailey, W. S. Keener, L. E. Davis, K. W. Herwig, K. Cekanova, Neutron imaging at the Oak Ridge National Laboratory: Application to biological research, in: Biomedical Science and Engineering Center Conference (BSEC), 2014 Annual Oak Ridge National Laboratory, IEEE, 2014, pp. 1-4. https://doi.org/10.1109/BSEC.2014.6867751

[8] H. Z. Bilheux, M. Cekanova, A. A. Vass, T. L. Nichols, J.-C. Bilheux, R. L. Donnell, V. Finochiarro, A novel approach to determine post mortem interval using neutron radiography, Forensic science international 251 (2015) 11-21. https://doi.org/10.1016/j.forsciint.2015.02.017

[9] L. Santodonato, H. Bilheux, B. Bailey, J. Bilheux, P. Nguyen, A. Tremsin, D. Selby, L. Walker, the CG-1D neutron imaging beamline at the Oak Ridge National Laboratory High Flux Isotope reactor, Physics Procedia 69 (2015) 104-108. https://doi.org/10.1016/j.phpro.2015.07.015

[10] Neutron Imaging at ORNL Home Page. https://neutronimaging.pages.ornl.gov. 
[11] Jupyter Notebooks. http://jupyter.org.

[12] Python Web Page. https://python.org

[13] Python notebook tutorial.

https://neutronimaging.pages.ornl.gov/tutorial/how_to_run_notebooks/

[14] thinlinc - a remote desktop server. https://www.cendio.com/thinlinc/what-is-thinlinc

[15] Conda environment. https://conda.io/docs/index.html.

[16] Garrett E. Granroth, K. An, H. L. Smith, P. Whitfield, J. C. Neuefeind, J. Lee, W. Zhou, V. N. Sedov, P. F. Peterson, A. Parizzi, H. Skorpenske, S. M. Hartman, A. Huq and D. L. Abernathy. Event-based processing of neutron scattering data at the Spallation Neutron Source, J. Appl. Cryst. (2018 51, 616-629. https://doi.org/10.1107/S1600576718004727 